\title{
DOES LOUD SOUND INFLUENCE THE INTRACOCHLEAR OXYGEN TENSION?
}

\author{
ALFRED L. NUTTALL ${ }^{1}$, ELISABETH HULTCRANTZ ${ }^{2}$ and MERLE LAWRENCE \\ 1 Department of Otorhinolaryngology, Kresge Hearing Research Institute, University of Michigan \\ Medical School, 1301 E. Ann Street, Ann Arbar, MI 48109, U.S.A., and ${ }^{2}$ Department of \\ Otorhinolaryngology, University Hospital, Uppsala, Sweden
}

(Received 10 March 1981 ; accepted 31 May 1981)

The effect of loud sound on the perilymphatic oxygen tension was studied in anesthetized guinea pigs. Pure tone $(4 \mathrm{kHz})$ and broad-band noise were given at $85-130 \mathrm{~dB}$ SPL for 3-8 min. No effects were seen either in the animals exposed to pure tone or in the animals exposed to $85 \mathrm{~dB}$ broad-band noise. In the animals exposed to noise at $130 \mathrm{~dB}$ SPL both increases and decreases of perilymphatic oxygen were measured but the changes were only of about $12 \%$ or less. The response to anoxia was normal. In animals with hypotension $(<8 \mathrm{kPa})$ the perilymphatic $P_{\mathrm{O}_{2}}$ fluctuated with the blood pressure. When the sound was delivered directly into the opened bulla the measured $P_{\mathrm{O}_{2}}$ dropped immediately but was found to be caused by the cooling effect of an air current produced by the noise. Flushing the opened bulla with nitrogen, air or oxygen caused the same temperature-induced drop of measured $P_{\mathrm{O}_{2}}$. The results and the artifacts are discussed.

Key words: guinea pig; perilymphatic oxygen tension; cochlear temperature; loud sound.

\section{INTRODUCTION}

The direct or indirect action of loud sound on inner ear microcirculation is thought to be a potential factor in causing dysfunction of the cochlea. Although it is clear that intense sound levels can mechanically disrupt the organ of Corti, more moderate sound levels may have multiple mechanical and metabolic effects. One possible way to study these effects is to measure alterations of the intracochlear oxygen tension. Oxygen tension can increase or decrease depending on the relative alteration of the source and sink relationships for oxygen.

Misrahy et al. [14] were the first to report loud sound effects on intracochlear $P_{\mathrm{O}_{2}}$ stating that the sound produced a large decrease in the endolymphatic $P_{\mathrm{O}_{2}}$. This result of their study has become deeply rooted in the literature of cochlear physiology. Subsequently a number of related studies seemed to give support to their finding. For example, inner-ear blood vessels appeared to be affected by loud sound $[5,10]$, and $\mathrm{PO}_{2}$ decreases were reported within the perilymph of scala tympani $[9,12]$.

In contrast to these findings, however, recently presented preliminary results by Nuttall et al. [17] indicated that the earlier measurements of $P_{\mathrm{O}_{2}}$ may have been incorrect. Noise and pure tones, with intensities up to $120 \mathrm{~dB}$ SPL for $5 \mathrm{~min}$, did not significantly alter the intracochlear $P_{\mathrm{O}_{2}}$ during the sound presentation or during a $30 \mathrm{~min}$ postexposure recording period and other recent and related studies seem to support these 
findings. For example, changes in the caliber of cochlear blood vessels due to sound exposure were not observed in studies by Vertes et al. [18] or Axelsson and Vertes [1], and loud sound did not result in a change in total cochlear blood flow [8].

The purpose of the present study was to expand the previous investigations by taking into account the blood pressure and cochlear temperature variables, thereby elucidating some of the pitfalls which may have caused the differences among the earlier investigators.

\section{MATERIALS AND METHODS}

Twenty-two guinea pigs weighing between 280 and $400 \mathrm{~g}$ with normal middle ears and normal Preyer reflex were used. Anesthesia was induced by premedication with Valium $(10 \mathrm{mg} / \mathrm{kg}$, i.m.) and Atropine $(0.125 \mathrm{mg} / \mathrm{kg}$, s.c.) followed $15 \mathrm{~min}$ later by Fentanyl (3.6 $\mathrm{mg} / \mathrm{kg}$ body wt., i.m.). The animals were tracheostomized and artificially ventilated. The respirator air was continuously examined for the content of $\mathrm{O}_{2}$ and $\mathrm{CO}_{2}$ and the respirator adjusted to give normal values. Constant body temperature of $38^{\circ} \mathrm{C}$ was secured by use of a heating pad. The arterial blood pressure was continuously recorded via a catheter placed into the right carotid artery. ECG and heart rate were recorded.

The animals were restrained supine with the head fixed in a headholder moveable in two planes. After a part of the mandible and the styloid process had been removed, the bulla was opened from an occipitolateral direction so the basal turn and the round window of the cochlea were clearly exposed. The ossicular chain and tympanic membrane were not disturbed. A small hole was drilled manually through the bony capsule into the scala tympani of the basal turn and, by means of a micromanipulator, an oxygen-sensitive electrode (Frederick Haer Co., model 30-05-2) was advanced towards the basilar membrane. The electrode penetrated through the hole to a depth of approximately 300 $\mu \mathrm{m}$ and did not contact the basilar membrane. The electrode was fixed in place by a carboxylate-type dental cement, preventing leakage of perilymph. After the oxygen current output from the electrode stabilized, and the magnitude of initial CM was determined, the animals were exposed to sound. All measurements of intracochlear oxygen were recorded as changes of the initial oxygen current and expressed as percent of initial $P_{\mathrm{O}_{2}}$.

For closed-field sound exposures, a sound speculum was fitted into the outer ear canal after the pinna had been removed. For free field sound exposures the speculum was 'aimed' in to the bulla opening. Sound was produced by a loudspeaker or by a $1 / 2$-inch condenser microphone and was calibrated with a probe-tube microphone. A wire electrode was placed onto the round window membrane so that the cochlear microphonic (CM) could be recorded. The ground electrode was placed in the neck muscles.

Six animals were exposed to a pure tone of $4000 \mathrm{~Hz}$ at $85 \mathrm{~dB} \mathrm{SPL}$ for $3 \mathrm{~min}$ and 30 min later they received another exposure at $105 \mathrm{~dB}$. Two animals received the same amount of sound exposure but white noise instead of pure tone. Five animals were exposed to a 3-8 min broad-band noise of approximately $130 \mathrm{~dB}$ SPL.

After each noise exposure the CM was measured for permanent threshold shift, and at the end of the experiment the respirator was turned off for $1.5 \mathrm{~min}$ so that the cochlear reaction to anoxia could be used to measure the sensitivity of the electrode to oxygen change. 


\section{Controls}

I. One guinea pig was exposed to sound via a bone-conducting sound device which was fitted on the upper teeth of the animal.

II. Four of the guinea pigs were exposed to loud noise $(130 \mathrm{~dB})$ in two ways: through the ear canal and before or after that exposure, also through the opening in the bulla. In three of these animals a thermocouple was placed on the round window membrane and the temperature was recorded before and during noise exposure.

III. In five of the animals different gases, nitrogen, air, or oxygen were flushed over the round window membrane while the perilymphatic $P_{\mathrm{O}_{2}}$ was measured. In one animal the temperature at the round window was also determined during the gas 'wash'.

\section{RESULTS}

All the animals tolerated the anesthesia and surgery with an initial mean arterial blood pressure above $9.0 \mathrm{kPa}$. The blood pressure, ranging between 9.0 and $13 \mathrm{kPa}$, was related to the age and weight of the animal with the higher values found in the animals over $400 \mathrm{~g}$. Normal values of $\mathrm{P}_{\mathrm{CO}_{2}}$ between 3.5 and 4.75 percent were measured for the exhalation air.

None of the animals exposed to pure tone of $4000 \mathrm{~Hz}$ showed any significant or consistent reaction of the $P_{\mathrm{O}_{2}}$ either when exposed to 85 or $105 \mathrm{~dB}$. The reaction to anoxia was, however, normal (see examples in Fig. 1). None of the animals exposed to white noise of 85 or $105 \mathrm{~dB}$ showed any reaction to the noise but two of them, which had

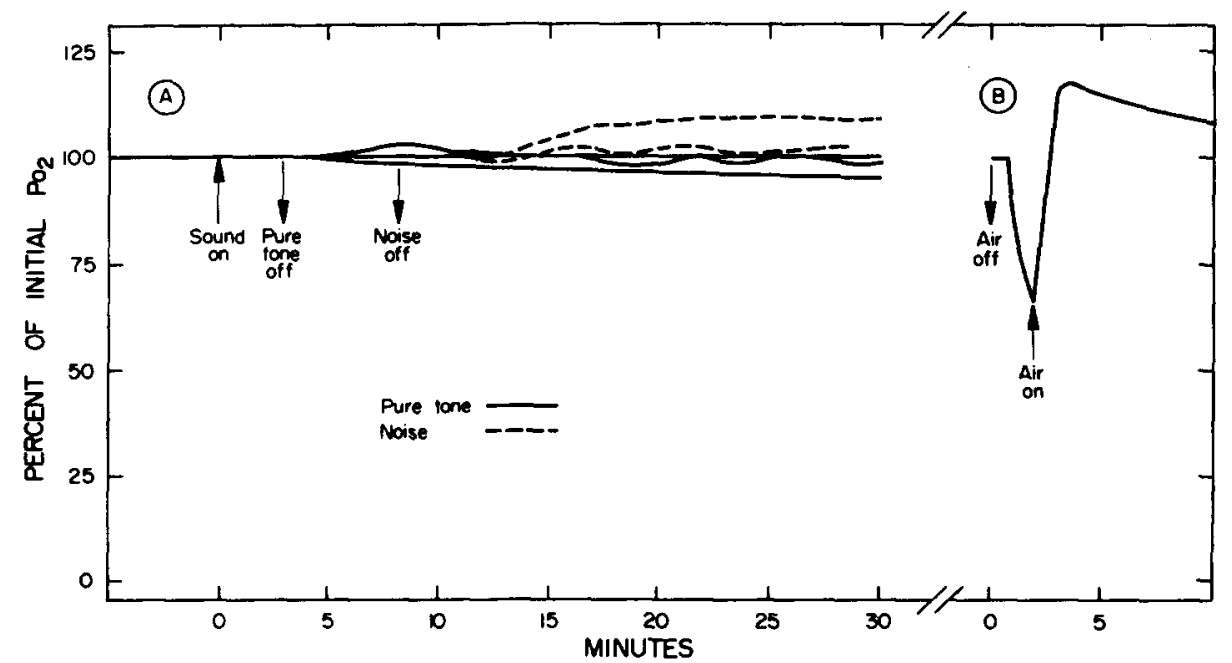

Fig. 1. (A) The effect of sound on oxygen tension in scala tympani perilymph of the basal turn. Three animals received a $3 \mathrm{~min}$ exposure to a $4 \mathrm{kHz}$ pure tone of $105 \mathrm{~dB}$ SPL (solid curves). Two animals were exposed to $130 \mathrm{~dB}$ of broad-band noise for $8 \mathrm{~min}$ (broken curves). The blood pressure was constant for these animals. (B) An example of the change in oxygen tension at the electrode location for a $1.5 \mathrm{~min}$ whole body anoxia. This was an in vivo test of the sensitivity of the electrode to oxygen change. 


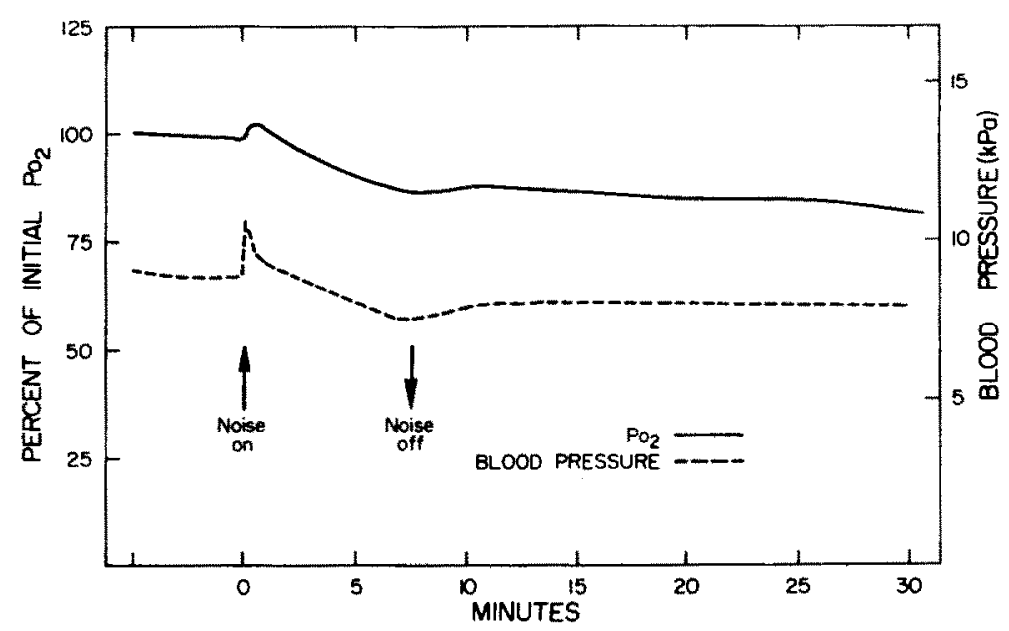

Fig. 2. A demonstration of the relationship between the change in the intracochlear oxygen tension (solid curve) and the change in the mean carotid blood pressure (broken curve) caused by a $7 \mathrm{~min}$ application of broad-band noise at $130 \mathrm{~dB}$ SPL. An interrelationship can occur when the blood pressure is below $8 \mathrm{kPa}$ (see text).

blood pressures around $8 \mathrm{kPa}$ or less, showed fluctuations in perilymphatic $P_{\mathrm{O}_{2}}$ which were related to the arterial blood pressure (see the example in Fig. 2). CM was slightly decreased after noise exposure. All the animals exposed to $130 \mathrm{~dB}$ broad-band noise demonstrated effects on their perilymphatic $P_{\mathrm{O}_{2}}$. In two animals a small increase (up to $12 \%$ ) of $P_{\mathrm{O}_{2}}$ was seen during the exposure (see Fig. 1). In two other cases a decrease of $P_{\mathrm{O}_{2}}$

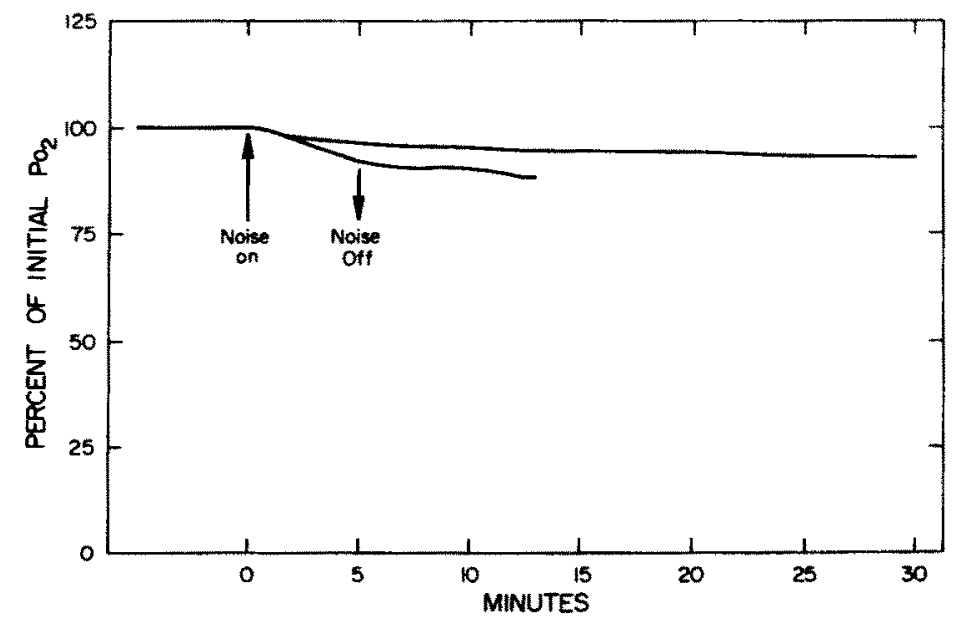

Fig. 3. Results from two animals in which the intracochjear $P_{\mathrm{O}_{2}}$ decreased during the bruad-band noise exposure of $130 \mathrm{~dB}$ SPL for $5 \mathrm{~min}$ and no recovery was observed during the recorded postexposure period. The blood pressure was constant for these animals. 


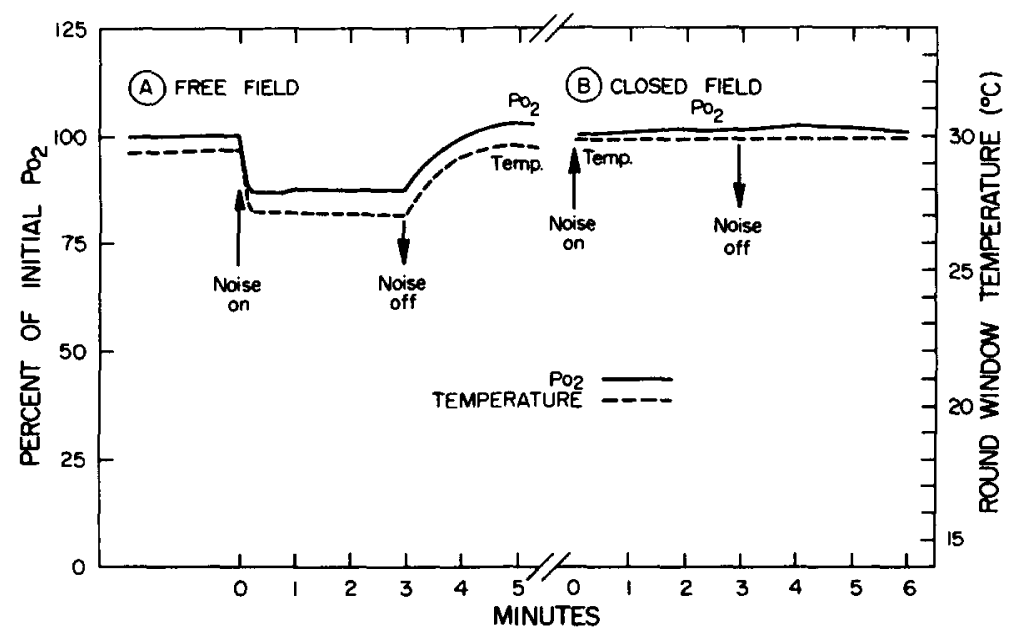

Fig. 4. Examples of the effect of a 3 min exposure of broad-band noise ( $130 \mathrm{~dB} \mathrm{SPL})$ on the intracochlear $P_{\mathrm{O}_{2}}$ (solid curves) and the round-window temperature (broken curves). (A) A free-field sound exposure where the sound tube was 'aimed' into the open bulla. (B) A closed-field sound exposure where the sound tube was sealed into the external meatus.

occurred during the exposure. The decrease stopped at the end of the exposure and no recovery could be seen within $30 \mathrm{~min}$ (see Fig. 3). The decrease was approximately $10 \%$ of the original value and much less than the decrease measured during whole body anoxia. During the noise exposure the $\mathrm{CM}$ was seriously affected, decreasing from approximately 1000 to $300 \mu \mathrm{V}$.

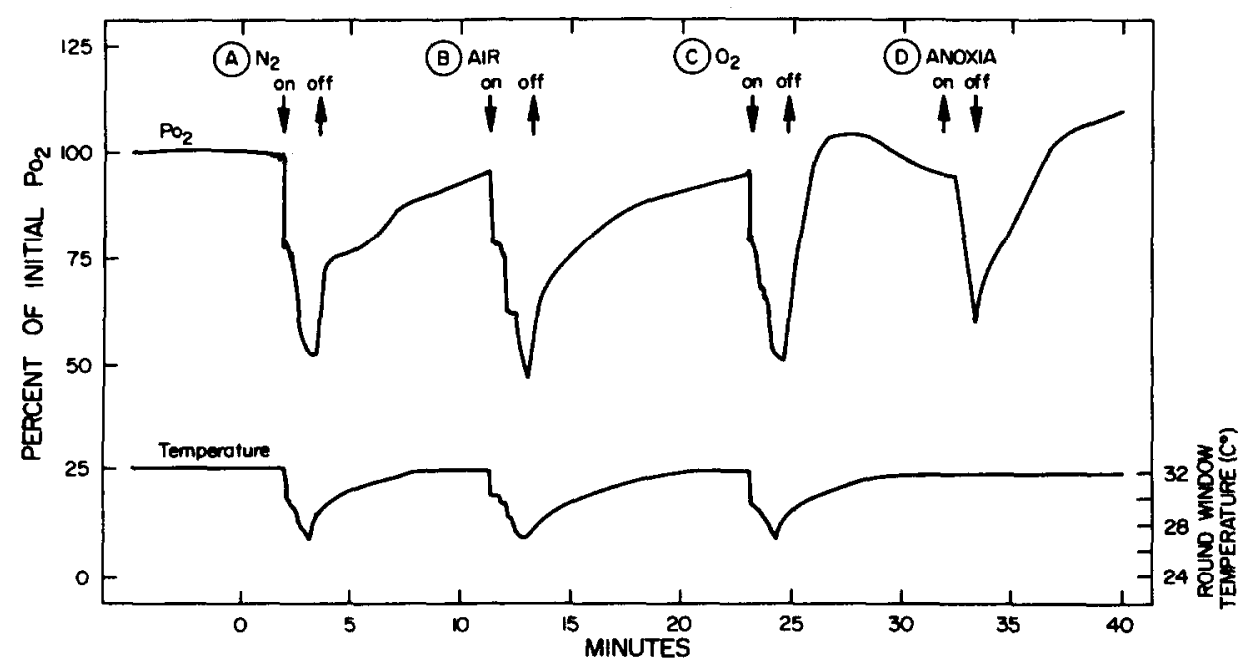

Fig. 5. The change in the intracochlear $P_{\mathrm{O}_{2}}$ (upper curve) and the round-window temperature (lower curve) caused by flushing various gases over the round window and by a whole body anoxia: (A) nitrogen; (B) air; (C) oxygen; (D) anoxia. 


\section{Controls}

$I$. No effect on the intracochlear $P_{\mathrm{O}_{2}}$ was seen in the animal exposed to approximately $90 \mathrm{~dB}$ white noise via bone conduction.

II. In all the animals which were exposed to noise by free field through the opening in the bulla an immediate drop in the $P_{\mathrm{O}_{2}}$ reading was noticed (Fig. 4A). The decrease in measured $P_{\mathrm{O}_{2}}$ was related to the drop in temperature which was recorded simultaneously. No decrease in $P_{\mathrm{O}_{2}}$ or temperature was registered when the sound was delivered via the ear canal (Fig. 4B).

III. All the animals which had their round windows flushed by gases showed a similar decrease of the recorded perilymphatic $P_{\mathrm{O}_{2}}$ irrespective of whether $\mathrm{N}_{2}$, air or $\mathrm{O}_{2}$ was used (see Fig. 5). The drop of measured $P_{\mathrm{O}_{2}}$ was related to the decrease in temperature (Fig. 5).

\section{DISCUSSION}

The pattern that emerges from the presented data suggests that low-level noise or puretone ( 85 and $105 \mathrm{~dB} \mathrm{SPL}$ ) exposures cause little or no perilymphatic $P_{\mathrm{O}_{2}}$ change (Fig. 1), while with high-level noise (130 dB SPL), there could be either a slight reduction or a slight increase in $P_{\mathrm{O}_{2}}$. When a reduction occurred (two animals), it was during the exposure period and the $P_{\mathrm{O}_{2}}$ level did not recover or decrease further after exposure (Fig. 2). This type of response might be consistent with mechanical damage to the organ of Corti because it occurs during the exposure and the cochlear microphonic is permanently reduced as well. When animals responded with increased $P_{\mathrm{O}_{2}}$ (two animals), it occurred after the intense noise exposure (Fig. 1) during the $30 \mathrm{~min}$ post-exposure period. This small increase in $P_{\mathrm{O}_{2}}$ may represent the beginning of a reactive hyperoxemia similar to that which was observed for a series of guinea pigs exposed to $4 \mathrm{~h}$ of noise and measured for $P_{\mathrm{O}_{2}}$ changes $2-7 \mathrm{~h}$ after exposure [17]. On the other hand, there is some evidence that exposure to noise leads to a decrease of the cochlear metabolism as determined by the deoxyglucose uptake procedure [21. With a constant blood flow to the cochlea during noise exposure [6] and a decreased utilization of oxygen, the decrease in metabolism might be reflected as an increase in available oxygen in perilymph.

The results presented above are in sharp contrast to the widely disseminated view that sound at levels as low as $85 \mathrm{~dB}$ SPL severely reduces the level of $P_{\mathrm{O}_{2}}$ in the perilymph or endolymph. That view is largely based on the results reported in studies by Misrahy et al. [14,15]. They report that pure tones of 130-135 dB SPL reduced endolymphatic $P_{\mathrm{O}_{2}}$ by $20-90 \%$ with full or partial recovery within $20 \mathrm{~min}$. With careful replication of their methods it was not possible to get the same results in the present study. However, many important parameters such as blood pressure and temperature were not mentioned in their report. The same objections can be applied to a similar study by Koide et al. [9].

Later Maass et al. [12,13] extended the study of sound effects on intracochlear $P_{\mathrm{O}_{2}}$ by measuring reductions in perilymphatic $P_{\mathrm{O}_{2}}$ in response to comparatively low-level (85 $\mathrm{dB}$ SPL) pink noise in the guinea pig and high-level pink noise in the cat (130 $\mathrm{dB})$. Perilymphatic $P_{\mathrm{O}_{2}}$ in scala tympani was observed to have a greater sensitivity to sound than endolymphatic $P_{\mathrm{O}_{2}}$. Although Maass and colleagues observed decreased $P_{\mathrm{O}_{2}}$ with 
sound, their results differ considerably from those of the earlier work by Misrahy and colleagues.

In the studies of Maass et al., low-level noise in guinea pig caused an immediate $P_{\mathrm{O}_{2}}$ reduction during the exposure period which then recovered to normal values within seconds after the exposure. High-level noise, in cat, did not seem to produce the immediate shift but rather a slow reduction and only after $30 \mathrm{~min}$ post-exposure was the $P_{\mathrm{O}_{2}}$ reduction significant.

Thus the results obtained in the two studies by Maass and colleagues do not repeat those of the earlier studies of Misrahy and colleagues and are not consistent within themselves if animal species differences are discounted.

Two experimental variables which may account for the data presented by Misrahy et al. and Maass et al. are blood pressure and cochlear temperature. In guinea pigs the blood pressure during different kinds of anesthesia is often low and decreasing [3]. In all the experiments of the present study, however, where there was little or no change in $P_{\mathrm{O}_{2}}$ during or after sound exposure, the mean arterial blood pressure was observed to be stable and greater than $9.0 \mathrm{kPa}$. The cochlea autoregulates its blood flow-oxygen supply in the same way as the brain but needs a blood pressure above approximately $8 \mathrm{kPa}$ for that purpose [7]. Near or below $8 \mathrm{kPa}$ the cochlear blood flow is directly correlated to the mean arterial blood pressure which was seen as fluctuations of the intracochlear $P_{\mathrm{O}_{2}}$ in the present study (Fig. 2). The loud sound caused the changes in the intracochlear $P_{\mathrm{O}_{2}}$ in these cases by its effects on the blood pressure. The drop in blood pressure during noise exposure seemed to be a vaso-vagal reflex, possibly elicited because of the light anesthesia used [6]. This evident connection between sound, blood pressure and intracochlear $P_{\mathrm{O}_{2}}$ stresses the importance of continuously monitoring blood pressure during this kind of experiment.

Temperature is another very important but little-controlled variable in the Misrahy and Maass studies. It is well known that the response of oxygen-sensitive electrodes is temperature dependent [4], and for absolute determinations of $P_{\mathrm{O}_{2}}$ the temperature of the calibration bath must match that of the experimental situation. It is often forgotten, however, that temperature may change during an experiment. Nuttall and LaRouere [16] have demonstrated the particular susceptibility of the guinea pig cochlea to temperature fall during anesthesia and surgery. In the present study it was discovered that it was possible to create temperature shifts in the guinea pig cochlea when loud free-field sound was transmitted to the open bulla from a sound tube connected to a loudspeaker. These were due to a sound-generated air flow, the so-called 'sonic wind' [11], which cooled the wet surfaces of the cochlea. Fig. 4A illustrates the 'apparent' $P_{\mathrm{O}_{2}}$ decreases that were closely related to the changing round-window temperature. Closed-field exposure did not produce the effect (Fig. 4B). Note that this artifact $P_{\mathrm{O}_{2}}$ shift caused by loud sound occurred, with fast time constants, during the sound exposure period.

Cooling of the cochlea is a variable which must be carefully controlled in any study of oxygen supply to the inner ear. As one control experiment, a nitrogen 'wash' was applied to the opened bulla in a similar manner as Maass et al. [12] to see how much of the intracochlear $P_{\mathrm{O}_{2}}$ was contributed by oxygen diffusion across the round window. A similar reduction in intracochlear $P_{\mathrm{O}_{2}}$ as Maass et al. obtained was observed, suggesting a role for the round window in diffusion of oxygen into perilymph. This effect, however, was also 


\section{TABLE I}

\section{CONTROL VARIABLES TESTED IN A PRELIMINARY STUDY USING 19 GUINEA PIGS}

No significant in tracochlear $P_{\mathrm{O}_{2}}$ change for loud sound (up to $120 \mathrm{~dB}$ SPL) was observed.

(a) Albino and pigmented guinea pigs were used.

(b) Urethane or Dial or Innovar anesthetics were used.

(c) Intracochlear oxygen tension and animal physiology were preconditioned by partial anoxia and hyperventilation.

(d) Oxygen electrode position:

(1) Perilymph of scala tympani in cochlear turns 1, 2 and 3.

(2) Endolymph of cochlear turns 1 and 2.

(e) Path of electrode penetration into scala media:

(1) Through the organ of Corti in the 'hook' area.

(2) Through the spiral ligament in the second cochlear turn.

(f) Pure tones of $100,200,500,1000,4000 \mathrm{~Hz}$ and broad-band noise were used as loud acoustic stimuli.

(g) Exposure times of $20 \mathrm{~s}$ to $5 \mathrm{~min}$ were used.

achieved during a 'wash' with air or pure oxygen and was subsequently demonstrated to be a temperature artifact (Fig. 5). Nevertheless, that the round window can have a diffusion role is suggested by the oxygen wash of Fig. 5 where the intracochlear $P_{\mathrm{O}_{2}}$ was increased over the pre-wash level when temperature had returned to the pre-wash value.

Several parameters other than blood pressure and temperature have been controlled and were presented in the preliminary report by Nuttall et al. [17]. These results are summarized in Table 1 . It is of particular importance that the results are not different if the electrode is placed into the endolymph of the scala media or in different turns of the guinea pig cochlea.

The conclusions from the present study are that moderate sound levels (up to at least $105 \mathrm{~dB}$ SPL noise or pure tone) do not affect the oxygen tension within the cochlea during a presentation of up to $8 \mathrm{~min}$ duration or in a $30 \mathrm{~min}$ post-exposure period, but destructive levels of sound (130 dB SPL) can cause small effects within these time periods.

\section{ACKNOWLEDGEMENTS}

This work was supported by Public Health Service Grants NS-05785, NS-15107 and NS-1 1731, the American-Scandinavian Foundation, and Thanks to Scandinavia, Inc.

\section{REFERENCES}

[1] Axelsson, A. and Vertes, D. (1980): Histological findings in cochlear vessels after noise. Proceedings of the Impulse Noise Conference, Syracuse, N.Y., June 1980. (in press).

[2] Canlon, B. and Schacht, J. (1981): The effect of noise on deoxyglucose uptake into inner ear tissues of the mouse. Abstract, 4th Midwinter Research Meeting, Association for Research in Otolaryngology, January 1981, St. Petersburg Beach, Fla. 
[3] Evans, E.F. (1979): Neuroleptanesthesia for the guinea pig. Arch. Otolaryngol. 105, 185-186.

[4] Fatt, I. (1976): Polarographic Oxygen Sensors. CRC Press, Cleveland, Ohio.

[5] Hawkins, J.E. (1971): The role of vasoconstriction in noise-induced hearing loss. Ann. Otol. Rhinol. Laryngol. 80, 903-913.

[6] Hultcrantz, E. (1979): The effect of noise on cochlear blood flow in the conscious rabbit. Acta Physiol. Scand. 106, 29-37.

[7] Hultcrantz, E., Linder, J. and Angelborg, C. (1977): Sympathetic effects of cochlear blood flow at different blood pressure levels. INSERM 68, 271-278.

[8] Hultcrantz, E., Angelborg, C. and Beausang-Linder, M. (1979): Noise and cochlear blood flow. Arch. Otolaryngol. 224, 103-106.

[9] Koide, Y., Yoshida, M., Konno, M., Nakano, Y., Yoshikawa, Y., Nagaba, M. and Morimoto, M. (1960): Some aspects of the biochemistry of acoustic trauma. Ann. Otol. 69, 661-697.

[10] Lawrence, M., Gonzalez, G. and Hawkins, J.E. (1967): Some physiological factors in noiseinduced hearing loss. J. Am. Ind. Hyg. Assoc. 28, 425-430.

[11] Lighthill, J. (1978): Waves in Fluids, pp. 337-351. Cambridge University Press, London.

[12] Maass, B., Baumgärtl, $H_{\text {. }}$ and Lübbers, D.W. (1976): Lokale $P_{\mathrm{O}_{2}}$ und pH Messungen mit Nadelelektroden zum Studium der Sauerstoffuersorgung und Mikrozirkulation des Innenohres. Arch. Otorhinolaryngol. 214, 109-124.

[13] Maass, B., Baumgärtl, $\mathrm{H}$. and Lübbers, D.W. (1978): Lokale $P_{\mathrm{O}_{2}}$ und $\mathrm{pH}_{2}$ Messungen mit Mikrokoaxialnadelelektroden an der Basalwidung der Katzencochlea nach akuter oberer zervikaler Sympathektomie. Arch. Otorhinolaryngol. 221, 269-284.

[14] Misrahy, G.A., Hildreth, K.M., Shinabarger, E.W., Clark, L.C. and Rice, E.A. (1958): Endolymphatic oxygen tension in the cochlea of the guinea pig. J. Acoust. Soc. Am. 30, 247-250.

[15] Misrahy, G.A., Shinabarger, E.W. and Arnold, J.E. (1958): Changes in cochlear endolymphatic oxygen availability, action potential, and microphonics during and following asphyxia, hypoxia, and exposure to loud sounds. J. Acoust. Soc. Am. 30, 701-704.

[16] Nuttall, A.L. and LaRouere, M.L. (1980): Depression of the guinea pig cochlear temperature caused by anesthesia and ventral approach ear surgery. J. Acoust. Soc. Am. 68, 489-493.

[17] Nuttall, A.L., Lawrence, M. and Burgio, P. (1979): Intracochlear oxygen tension during and after loud sound exposure. J. Acoust. Soc. Am. 65, Suppl. 1, S12 (Abstract).

[18] Vertes, D., Axelsson, A. and Lipscomb, D.M. (1979): Some vascular effects of noise exposure in the chincilla cochlea. Arch. Otorhinolaryngol. 224, 97-101. 